\title{
Investigation of Single Slotted Cylinder Placed in a Fin Tube Heat Exchanger Model
}

\author{
[Sedat Yayla, Besir Sahin, Suat Onal, Huseyin Akıllı, Suleyman Teksin]
}

\begin{abstract}
The flow characteristics of inline and one slotted cylinder placed in a fin tube heat exchanger model are experimentally investigated using Partical Image Velocimetry (PIV) for Reynolds numbers of 1500 and 4000. In this study, the Reynolds number is calculated based on the cylinder diameter. Qualitative flow visualization was initially employed in order to observe the overall nature of the vortical flow patterns of cylinders. The flow interactions in the streamwise bases of the cylinder with a flat plate surface results in two dimensional vorticity $\langle\omega\rangle$, streamline topology $\langle\psi\rangle$ and velocity vector map $\langle V\rangle$ behavior.
\end{abstract}

Keywords - confined flow, vorticity, staggered cylinders, slot cylinders

\section{Introduction}

A heat exchanger is a device which transfers heat energy from one fluid (or gas) to another fluid (or gas), and without mixing the two different fluids (or gases). A common example is automotive radiators. Heat from the hot engine water is pumped through the radiator, while air is blown through the radiator tins. The hot engine water's heat energy is transferred to the air, thus keeping the water at the right temperature, and thus the engine is kept at the right temperature. Essentially an automotive radiator is a Liquid to Air Heat Exchanger.

In the plate fin and tube heat exchangers, continuous and plain or specially configured fins are used on the outside of the array of tubes of staggered or in-lined arrangement passing perpendicularly through the plates to improve the heat transfer coefficient on that side. Heat exchangers, generally plain fins are used because of their long term operation and lower friction characteristics. Similarly, generally round tubes are preferred because of their easy construction. Therefore some investigators such as Mon and Gross (2004), Kim and Song (2002), Tiwari at al., (2003), Saboya and Sparrow, (2001), Bouris at al., (2001), Méndez at al. (2000), Bouris and Bergeles, (1999), Jang at al., (1997), Tsai and Sheu, (1998), used elliptical and round tubes in their investigations.

Sedat Yayla, Suleyman Teksin

Yuzuncu Yil University

Turkey

Besir Sahin, Huseyin Akilli

Cukurova University

Turkey

Suat Onal

Korkut Ata University

Turkey
One of the main problems in the fluid mechanics is to determine the flow characteristics around bluff bodies and especially cylinders. Of course, formation and shedding of vortices from a cylinder have fundamental research values in the areas of vortex dynamics and bluff body flows. Nakamura (1996) has investigated vortex shedding and vortex shedding frequency experimentally for different type of bluff bodies which are rectangular, semi-circular and circular with splitter plates in a wind tunnel. He reported that when increasing the length and diameter of pipe ratio, $\mathrm{L} / \mathrm{D}$, the strouhal number of a bluff body decreases, the reduction not being dependent on the details of the cross-sectional geometry but only the value of $\mathrm{L} / \mathrm{D}=1.0$ Zdravkovich (1988) reported overall idea about different modes of vortex shedding. He categorized the observed modes of vortex shedding into 3 groups. The first one is the low-speed mode caused by the mechanics of vortex formation and shedding. Second is the synchronized vortex shedding. Third one is another synchronized vortex shedding is described that is caused by the streamwise oscillation of a cylinder or when a stationary cylinder is submerged in oscillatory flows.

Sumner et al. (2000) investigated flow characteristics behind side-by-side cylinders using PIV technique. Dong and Karniadakis (2005) presented results of direct numerical simulations for turbulent flow past a stationary and a rigid circular cylinder for $\mathrm{Re}=10000$ and compared their results with available experimental data.

\section{Experimental Set Up and Instrumentation}

In the present study, qualitative flow visualization was employed during the initial scope of experiments in order to determine the overall flow patterns for a circular cylinder of $50 \mathrm{~mm}$ diameter, a circular slotted-cylinder of $50 \mathrm{~mm}$ diameter and $5 \mathrm{~mm}$ slot's width mounted over a flat plate and also located between two parallel plates. Figure 1 shows general description of the test geometry of single slotted-cylinder in plan view plane.

In order to determine the instantaneous velocity fields around the surface mounted cylinders in the heat exchanger flow passages, high-image-density Particle Image Velocimetry (PIV) technique was employed. 


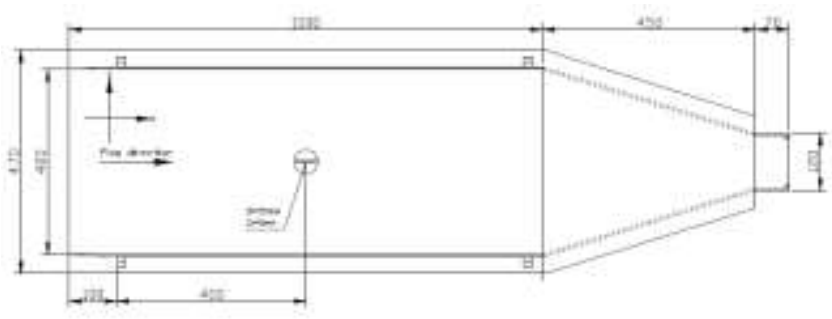

Figure 1. Test geometry of single slotted-cylinder in plan view plane (Dimensions in $\mathrm{mm}$ )

Particle Image Velocimetry (PIV) is a nonintrusive measurement technique used to simultaneously determine the velocities at many points in a fluid flow. The technique involves seeding the flow field, illuminating the region under investigation and capturing two images of that region in rapid succession. The general principle of PIV is to illuminate tracer particles in the flow field of interest with a plane sheet of light, and acquire two images of the flow field with a known time separation. When a digital CCD camera is used for image acquisition, one uses the acronym DPIV. The displacement field is determined from the motion of the tracer particles between the two images, and by dividing this with the known time separation one obtains the velocity field.

Particle size and density, and fluid density and viscosity, determine the effects of buoyancy and inertia. Exact neutral buoyancy is difficult to achieve, but particles must remain suspended throughout an experiment. In general, seeding is easier in fluids. Lower velocities and accelerations combined with the higher density and viscosity of liquid means larger, more easily detectable images.

In PIV, the displacement field is determined as average displacements within so-called interrogation areas of the image plane. A typical size of these interrogation areas is $32 \times 32$ pixels, which means that one gets about 1000 vectors from an image with a resolution of $1008 \times 1016$ pixels. For single exposed images, the displacement is determined by forming the crosscorrelation of corresponding interrogation areas in the first and second images.

For the illumination, it is preferable to use a laser, since the laser beam is easy to form into a sheet by a cylindrical lens. A pulsed laser is preferred, since one obtains a high light energy during a very short time interval (typically $5 \mathrm{~ns}$ for a YAG-laser), which means that the particle images will be practically frozen even for high velocities $(>100 \mathrm{~m} / \mathrm{s})$. The repetition rate of a YAGlaser is typically $10-30 \mathrm{~Hz}$, which is too low except for very low velocities $(<1 \mathrm{~cm} / \mathrm{s})$. One therefore needs two lasers to get full freedom in terms of time separation between the pulses. Special PIV YAG-lasers are available that combine two laser cavities with a common beam outlet.

Experiments were performed in a large-scale water channel, having a test section length of $650 \mathrm{~mm}$ and width $750 \mathrm{~mm}$. In order to study the case of a confined flow, the test section, which has a total height of $610 \mathrm{~mm}$ was filled with water. In order to characterize the flow structure downstream of single slotted-cylinder.

To be able to acquire two single exposed images with a time separation of the order of microseconds, one uses a so-called full-frame interline transfer progressive scan CCD camera, also called a cross-correlation CCDcamera. The basic idea is that the image exposed by the first laser pulse is transferred very rapidly to light-hidden areas on the CCD-chip.

\section{Result and Discussion}

The objective of the present work is to conduct quantitative studies in plan-view laser planes for a circular cylinder, a circular slot-cylinder, multiple circular cylinders and multiple circular slot-cylinders which are mounted on a flat plate for the purpose of revealing the structure of the flow details using instantaneous and timeaveraged flow data. Plan-views of the test section, direction of the flow and the coordinate system are all illustrated in Figure 2. The dimensions of the base plate are $1000 \mathrm{~mm} \times 400 \mathrm{~mm} \times 100 \mathrm{~mm}$. The diameter and the submerged part of the cylinder are $\mathrm{d}=50 \mathrm{~mm}$ and $\mathrm{h}=20 \mathrm{~mm}$, respectively.

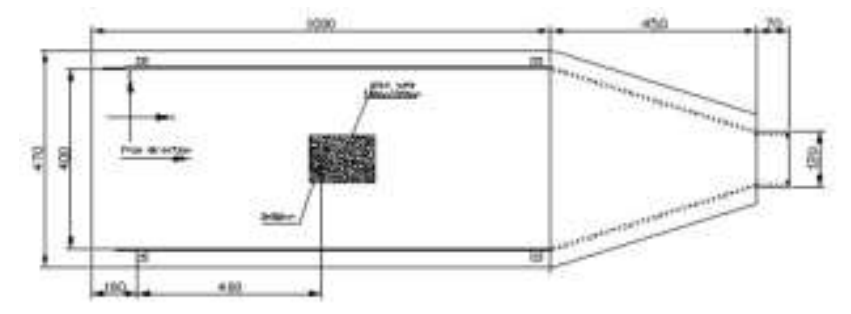

Figure 2. Test geometry, size of images, location of measuring planes in plan view plane (Dimansions in $\mathrm{mm}$ ).

Examing figure 3 and 4 reveal that having $5 \mathrm{~mm}$ slot at central line of the circular cylinder vortical flow behavior around the slotted-cylinder is completely changed. the saddle point in the upstream region of the slotted-cylinder move close to the forward face of the cylinder for both cases of Reynolds numbers. In addition, vortices travels in the free stream flow direction close to the outer surface of the shedding vortices. Having flow jet through the slot a wake flow region of the cylinder is further activated. 
Proc. of the Second Intl. Conf. on Advances in Mechanical and Automation Engineering - MAE 2015

Copyright (C) Institute of Research Engineers and Doctors, USA .All rights reserved.

ISBN: 978-1-63248-045-3 doi: 10.15224/ 978-1-63248-045-3-39

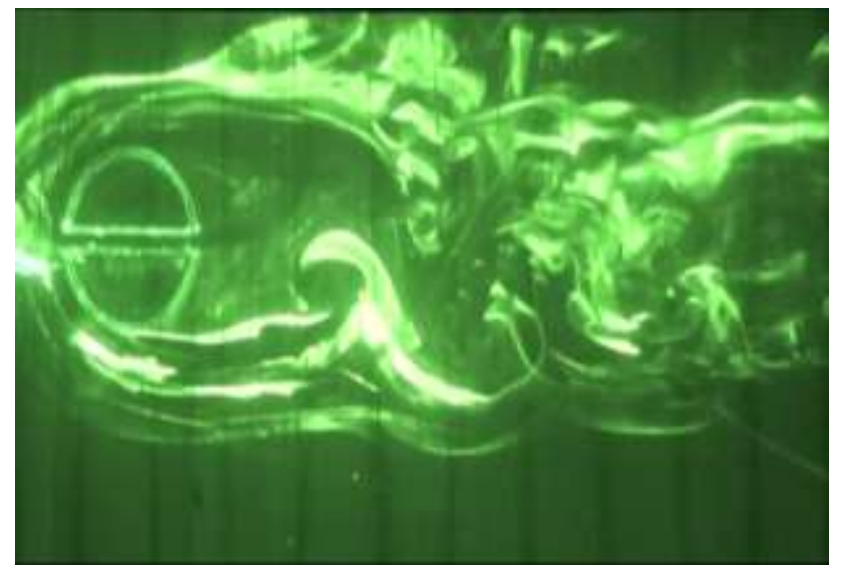

Figure 3. Instantaneous dye visualization of single slot-cylinder for $\mathrm{Re}_{\mathrm{d}}$ $=1500, \mathrm{~h}_{\mathrm{L}} / \mathrm{h}_{\mathrm{W}}=0.1$

Confined flow past a cylinder gives rise to a vortex about the upstream surface of the cylinder, and large-scale vortex formation occurs in the near-wake. The consequence of a streamwise slot through the cylinder is used to alter the patterns of the flow structure along the fore and base regions of the cylinder, as well as in its nearwake. Such alterations have important implications, not only for the steady and unsteady loading on the cylinder, but also the corresponding loading on the bed region about the cylinder.

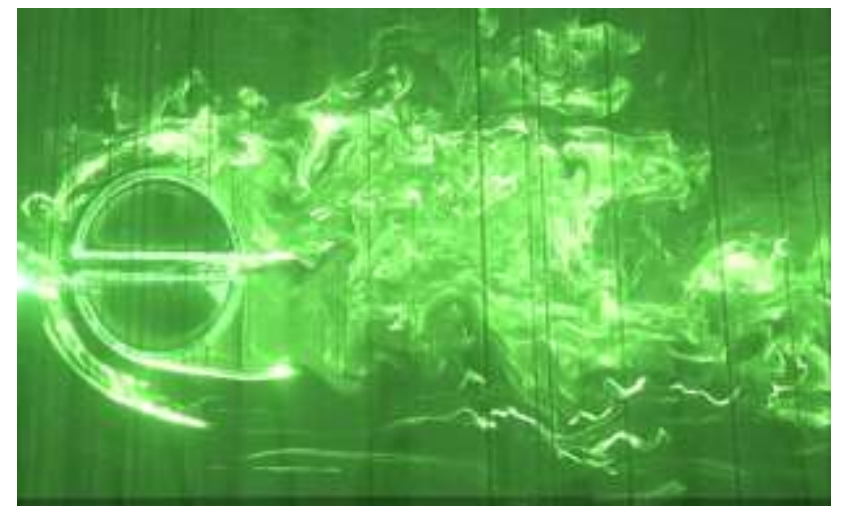

Figure 4. Instantaneous dye visualization of single slot-cylinder for $\mathrm{Re}_{\mathrm{d}}$ $=4000, \mathrm{~h}_{\mathrm{L}} / \mathrm{h}_{\mathrm{W}}=0.1$

During the dye visualization it is seen that the vortex that exists about the upstream surface of the complete cylinder or without a slot is altered significantly due to the flow extraction through a slot.
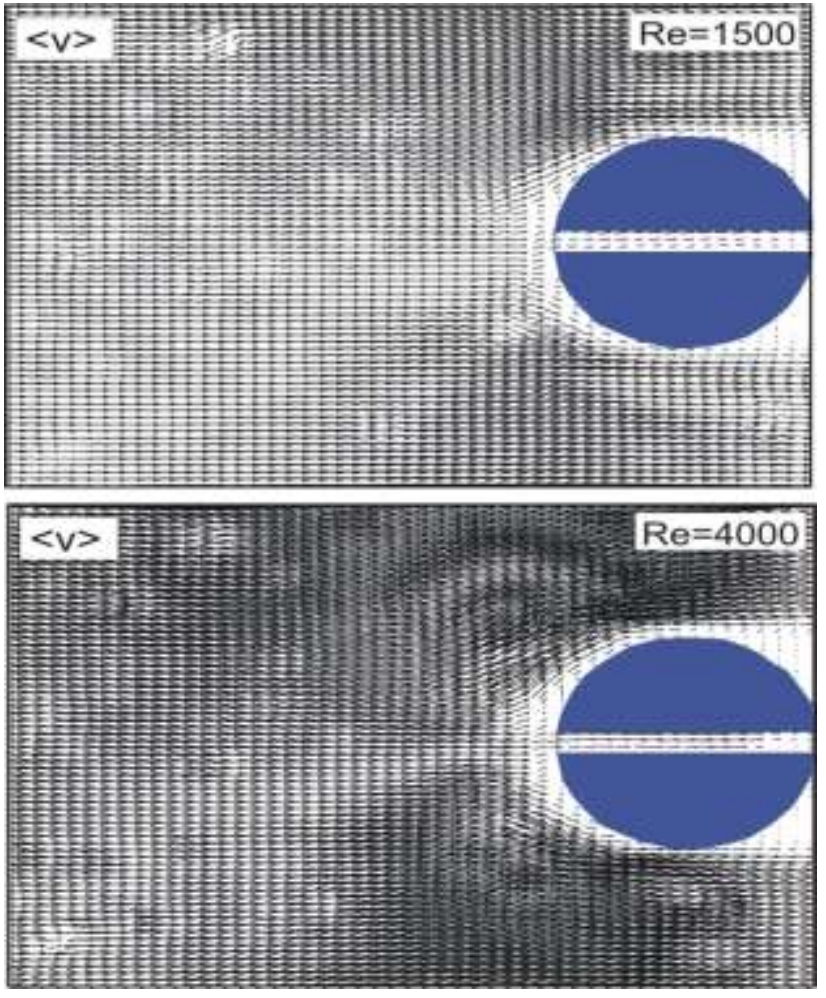

Figure 5. Time-averaged velocity vector map in front of single slotcylinder for $h_{L} / h_{W}=0.1, \operatorname{Re}_{d}=1500$ and 4000

Figure 5 shows time-averaged velocity vector map at $2 \mathrm{~mm}$ above the base of the cylinder, for $\mathrm{Re}=1500$ and $\operatorname{Re}=4000$. The size of the separation is obtained symmetric in both Reynolds numbers and slightly wide at $\mathrm{Re}=4000$ comparing with $\mathrm{Re}=1500$ 
Proc. of the Second Intl. Conf. on Advances in Mechanical and Automation Engineering - MAE 2015

Copyright (C) Institute of Research Engineers and Doctors, USA .All rights reserved.

ISBN: 978-1-63248-045-3 doi: 10.15224/ 978-1-63248-045-3-39
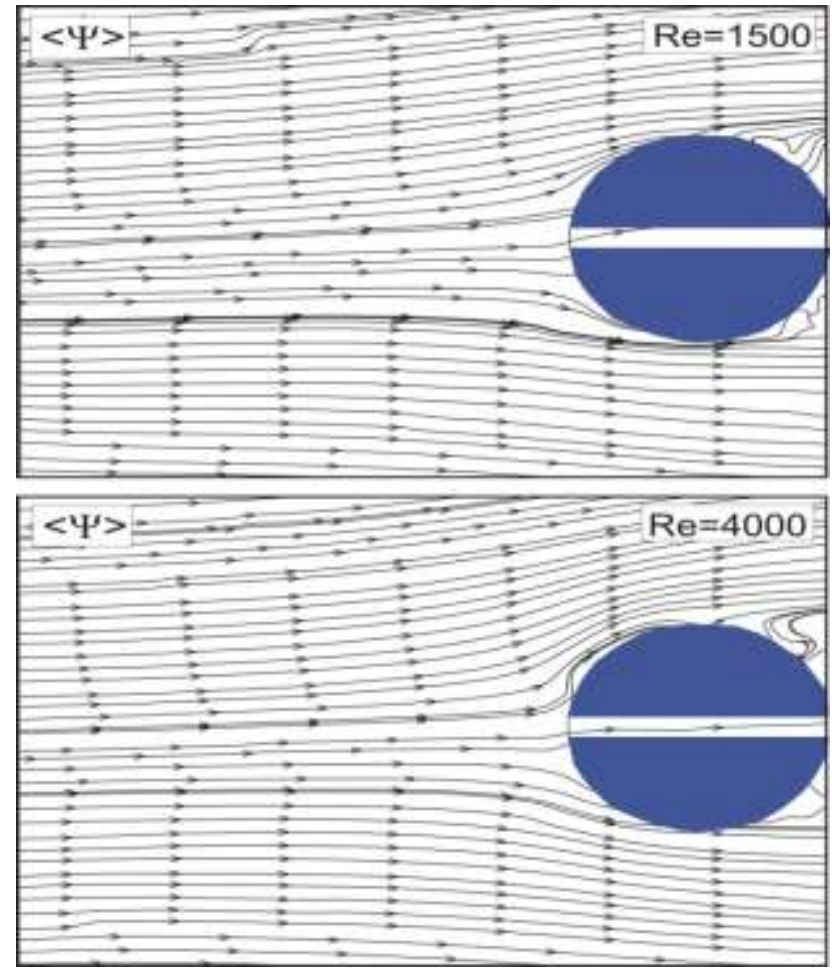

Figure 6. Time-averaged streamline pattern in front of single slotcylinder for $\mathrm{h}_{\mathrm{L}} / \mathrm{h}_{\mathrm{W}}=0.1, \mathrm{Re}_{\mathrm{d}}=1500$ and 4000

Figure 6 illustrates time-averaged streamline pattern at $2 \mathrm{~mm}$ above the base of the cylinder, for $\mathrm{Re}=7000$. It is clearly seen that wide and symmetrical two foci rotating in the opposite direction appears in the wake region. A saddle point followed by these two symmetrical foci is also observed.

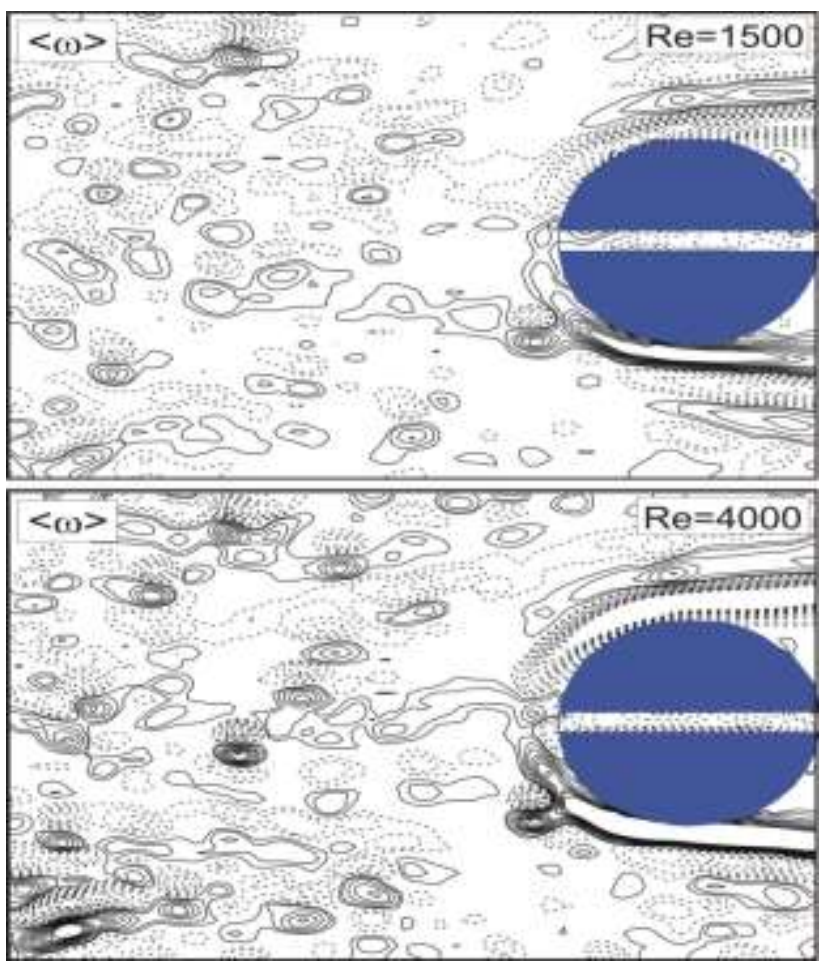

Figure 7. Time-averaged vorticity contours in front of single slot cylinder for $\mathrm{h}_{\mathrm{L}} / \mathrm{h}_{\mathrm{W}}=0.1, \mathrm{Re}_{\mathrm{d}}=1500$ and $4000 . \omega= \pm 0.5 \mathrm{~s}^{-1}$ and $\Delta \omega=1 \mathrm{~s}^{-1}$

Figure 7 demonstrates time-averaged vorticity contours at $2 \mathrm{~mm}$ above the base of the cylinder, at $\mathrm{Re}=1500$ and $\mathrm{Re}=4000$. It is clearly seen that the vorticity layers beginning from the stagnation point of the cylinder are convecting in the flow direction in the shear layer. It is observed that the vorticity layers are symmetric with each other according to the plane of symmetry of the cylinder. The positive vorticity layers and the negative vorticity layers are drawn as solid and dashed lines, respectively. The minimum and the incremental values of the vorticity are $\omega= \pm 0.5 \mathrm{~s}^{-1}$ and $\Delta \omega=1 \mathrm{~s}^{-1}$, respectively
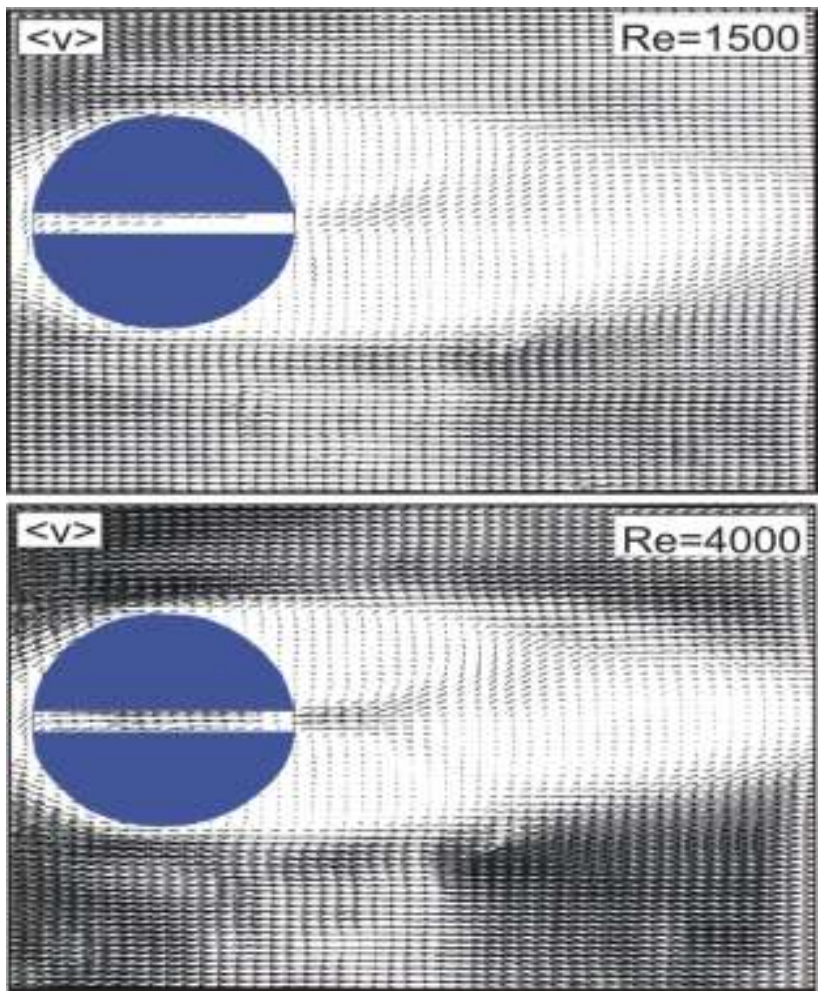

Figure 8. Time-averaged velocity vector map in wake flow of single slot-cylinder for $h_{L} / h_{W}=0.1, \operatorname{Re}_{d}=1500$ and 4000

Figure 8 shows time-averaged velocity vector map of wake flow at $2 \mathrm{~mm}$ above the base of the cylinder, for $\operatorname{Re}=1500$ and $\mathrm{Re}=4000$. The size of the wake region is obtained wide and two symmetric foci rotating in opposite direction are clearly seen.

Streamline topology, $\langle\psi\rangle$, show that flow occurred due to the hydrodynamics of the vortex system and from the slot is shifted in to the wake flow regions for forward field. The corresponding streamline topology reveal that symmetrical foci with opposite circulation and saddle points downstream are evident as indicated in figure 9 . 
Proc. of the Second Intl. Conf. on Advances in Mechanical and Automation Engineering - MAE 2015

Copyright (C) Institute of Research Engineers and Doctors, USA .All rights reserved.

ISBN: 978-1-63248-045-3 doi: 10.15224/ 978-1-63248-045-3-39
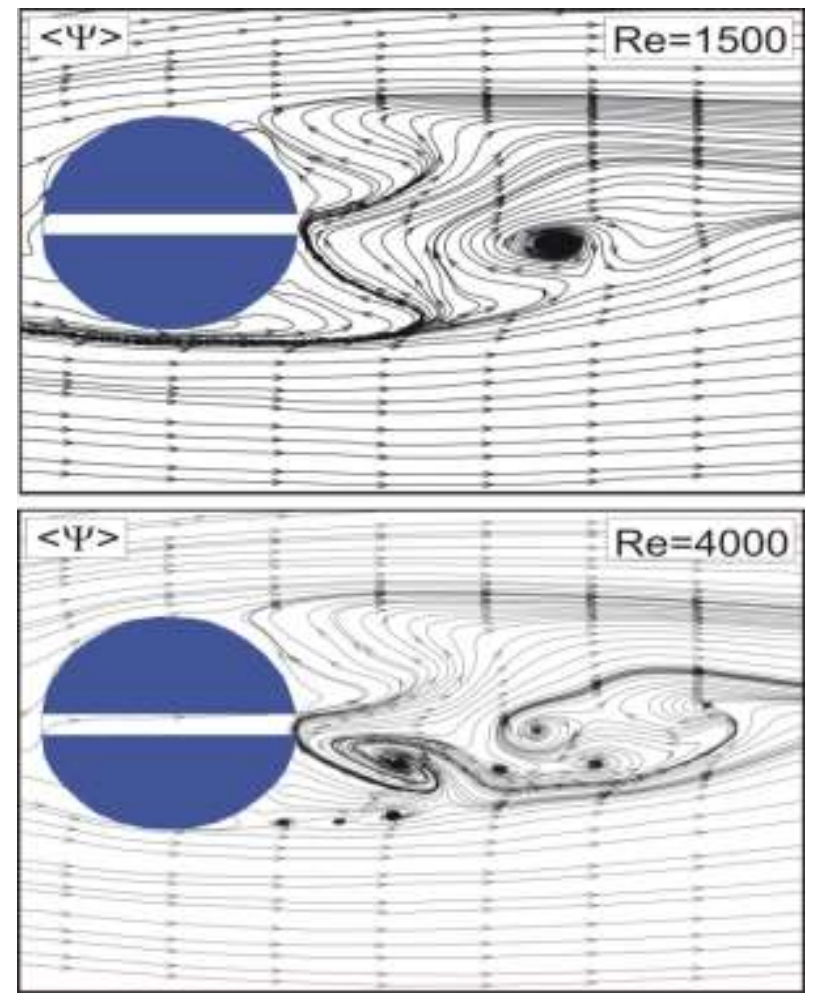

Figure 9. Time-averaged streamline pattern in wake flow of single slotcylinder for $\mathrm{h}_{\mathrm{L}} / \mathrm{h}_{\mathrm{W}}=0.1, \mathrm{Re}_{\mathrm{d}}=1500$ and 4000
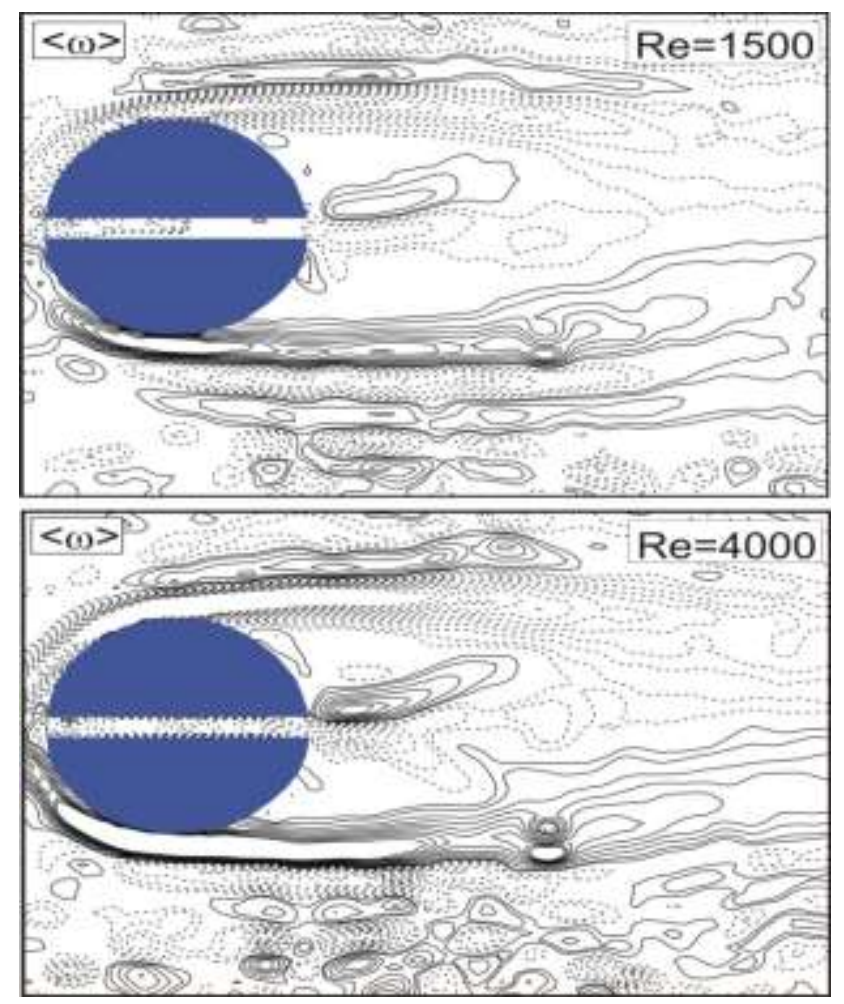

Figure 10. Time-averaged vorticity contours in wake flow of single slotcylinder for $h_{L} / h_{W}=0.1, R_{d}=1500$ and $4000 . \omega= \pm 0.5 \mathrm{~s}^{-1}$ and $\Delta \omega=1 \mathrm{~s}^{-1}$

Figure 10 demonstrates time-averaged vorticity contours at $2 \mathrm{~mm}$ above the base of the cylinder, at $\mathrm{Re}=1500$ and $\mathrm{Re}=4000$. It is clearly seen that the vorticity layers beginning from the stagnation point of the cylinder are convecting in the flow direction in the shear layer. It is observed that the vorticity layers are symmetric with each other according to the plane of symmetry of the cylinder. The positive vorticity layers and the negative vorticity layers are drawn as solid and dashed lines, respectively. The minimum and the incremental values of the vorticity are $\omega= \pm 0.5 \mathrm{~s}^{-1}$ and $\Delta \omega=1 \mathrm{~s}^{-1}$, respectively.

\section{Conclusion}

Heat exchangers, which are used in wide variety of industrial applications, are one of the devices that can be developed to energy saving, from the hydrodynamics manner. Therefore the main purpose of the present study is to investigate details of flow structure of a circular single slotted cylinder cylinders mounted on a flat surface in a narrow gaped rectangular duct in the case of confined flow which is used in plate-fin-and-tube type of heat exchangers.

The width of the slot is $5 \mathrm{~mm}$, the diameters of the cylinders are $50 \mathrm{~mm}$ and the ratio of $\mathrm{B} / \mathrm{D}$ is 0.1 , the gap ratio $G / D$ is 2 where the slot width is $B$, diameter of the cylinder is D. The laser sheet was located parallel to the bottom surface of the water channel at the elevation of $\mathrm{hl} / \mathrm{hw}=0.1$. The slotted-cylinders significantly change the flow characteristics in downstream directions.

During the experiments, Reynolds numbers were changed between 1500 and 4000. The cylinders having same slot widths were used during the experiments. All experiments were carried out at elevation of $h_{1} / h_{w}=0.1$.

Following conclusions can be derived from the overall experimental studies:

- The heat transfer rate increases with an increase of the Reynolds number.

- The size of the wake region differs slightly depending on the Reynolds number for single slotted-cylinder

- The positive and the negative vorticity layers are approximately equal and symmetric with each other.

- The slot width has an effect on the flow characteristics.

\section{References}

[1] M. S.R. Mon, U. Gross, "Numerical study of fin-spacing effects in annular finned tube heat exchangers," International Journal of Heat and Mass Transfer, 47, 1953-1964, 2004.

[2] J.Y. Kım, T. H. Song, "Microscopic Phenomena and Macroscopic Evaluation of Heat Transfer From Plate Fins/Circular tube Assembly Using Naphthalene Sublimation Technique," International Journal of Heat and Mass Transfer, 45: 3397-3404, 2002.

[3] S. Twari, G. Biswas, and P.L. N. Prasad Sudıpta Basu, "Numerical Prediction of flow and heat transfer in a rectangular channel with a build-in circular tube," Transactions of the ASME, Journal of Heat Transfer, 125, 413-421, 2003.

[4] D. Bourıs, G. Papadakis, G.Bergeles, "Numerical Evaluation of Alternate Tube Configurations for Particle Deposition Rate Reduction in Heat Exchanger Tube Bundles," International Journal of Heat and Fluid Flow, 22, 525-536, 2001. 
[5] R. R. Mendez, M. Sen, K. T. Yang, R. Mcclain, "Effect of Fin Spacing on Convection in a Plate Fin and Tube Heat Exchanger," Int. J. Heat Mass Transfer, 43: 39-51, 2000.

[6] J. Y. Jang, L. K. Chen, "Numerical Analysis of Heat Transfer and Fluid Flow in a Three- Dimensional Wavy Fin and Tube Heat Exchanger,'International Journal of Heat and Mass Transfer, 40(16): 3981-3990, 1997.

[7] S. F. Tsa1, T. W. H. Sheu, "Some Physical Insights into a TwoRow Finned Tube Heat Transfer," Computers and Fluids, 27(1): 29-46, 1998.

[8] Y. Nakamura, "Vortex shedding from bluff bodies and a universal Strouhal Number," Fluids and Structures Volume 10, Issue 2, Pages 159-171, 1996.

[9] M. M. Zdravkovich, "Review of interference-induced oscillations in flow past two parallel circular cylinders in various arrangements," J. Wind Ind. Aerodyn. 28 183-200, 1988.

[10] D. Sumner, D., S. J. Price, And M. P. Paidoussis, "Flow-pattern identification for two staggered circular cylinders in cross-flow," Journal of Fluid Mechanics, 411: 263-303, 2000.

[11] S. Dong and G.E. Karniadakis, "DNS of flow past a stationary and oscillating cylinder at $\mathrm{Re}=10000$, , Journal of Fluids \& Structures, vol. 20(4), pp. 519-531, 2005.

[12] D. Bouris, G. Bergeles, "Two dimensional time dependent simulation of the subcritical flow in a staggered tube bundle using a subgrid scale model," Int. J. Heat Fluid Flow 20 (2), 105-114, 1999. 